ARTICLE

Received 24 Feb 2013 | Accepted 24 May 2013 | Published 19 Jun 2013

DOI: $10.1038 /$ ncomms 3053

\title{
Magnetoelectric effects and valley-controlled spin quantum gates in transition metal dichalcogenide bilayers
}

Zhirui Gong ${ }^{1,2, \star}$, Gui-Bin Liu ${ }^{1,2,3, \star}$, Hongyi Yu $u^{1,2}$, Di Xiao ${ }^{4}$, Xiaodong Cuil, Xiaodong Xu ${ }^{5,6} \&$ Wang Yao ${ }^{1,2}$

In monolayer group-VI transition metal dichalcogenides, charge carriers have spin and valley degrees of freedom, both associated with magnetic moments. On the other hand, the layer degree of freedom in multilayers is associated with electrical polarization. Here we show that transition metal dichalcogenide bilayers offer an unprecedented platform to realize a strong coupling between the spin, valley and layer pseudospin of holes. Such coupling gives rise to the spin Hall effect and spin-dependent selection rule for optical transitions in inversion symmetric bilayer and leads to a variety of magnetoelectric effects permitting quantum manipulation of these electronic degrees of freedom. Oscillating electric and magnetic fields can both drive the hole spin resonance where the two fields have valley-dependent interference, making an interplay between the spin and valley as information carriers possible for potential valley-spintronic applications. We show how to realize quantum gates on the spin qubit controlled by the valley bit.

\footnotetext{
${ }^{1}$ Department of Physics, The University of Hong Kong, Hong Kong, China. ${ }^{2}$ Center of Theoretical and Computational Physics, The University of Hong Kong, Hong Kong, China. ${ }^{3}$ School of Physics, Beijing Institute of Technology, Beijing 100081, China. ${ }^{4}$ Department of Physics, Carnegie Mellon University, Pittsburgh, Pennsylvania 15213, USA. ${ }^{5}$ Department of Physics, University of Washington, Seattle, Washington 98195, USA. ${ }^{6}$ Department of Material Science and Engineering, University of Washington, Seattle, Washington 98195, USA. ${ }^{\star}$ These authors contributed equally to the work. Correspondence and requests for materials should be addressed to W.Y. (email: wangyao@hku.hk).
} 
$\mathrm{D}$ evice applications exploiting internal degrees of freedom of charge carriers may lead to new electronic technologies outperforming their conventional counterparts, which rely on charge flow only. A seminal example is electron spin, which has been extensively exploited as a carrier of classical or quantum information ${ }^{1-3}$. Recently, the emergence of atomically thin two-dimensional (2D) crystals such as graphene and transition metal dichalcogenides (TMDC) have offered new playgrounds to explore novel electronic device concepts ${ }^{4-10}$. In addition to spin, two types of internal indices of electrons have been investigated as information carriers, namely the layer ${ }^{11-14}$ and valley ${ }^{15-19}$ degrees of freedom.

The layer degree of freedom exists in bilayer systems and can be described as a pseudospin. Pseudospin up (down) refers to the state where the charge carrier is located in the upper (lower) layer. Thus, pseudospin polarization directly corresponds to electrical polarization. Such layer pesudospin has been proposed in graphene bilayers for pseudospintronics ${ }^{11-14}$. Valley refers to the degenerate extrema of energy bands, which in many hexagonal 2D crystals are located at the corners of the hexagonal Brillouin zone ( $K$ points). It was predicted that in the absence of inversion symmetry valley degree of freedom can be associated with magnetic moment and optical circular dichroism ${ }^{18,20}$, which has made possible the first observations of optical pumping of valley polarization in TMDC monolayers $^{21-23}$ and biased bilayers ${ }^{24}$. As the spin and valley have magnetic moment that can be controlled by magnetic and optical means, while the layer pseudospin corresponds to an electrical polarization subject to electrical manipulation, a system allowing interplay of these different degrees of freedom may offer unprecedented possibilities to exploit their quantum control for new device concepts.

In this letter, we show that in group-VI TMDC bilayers, the spin, the layer pseudospin and the valley degrees of freedom are strongly coupled for the holes in the $K$ valleys, leading to a variety of magnetoelectric effects to realize valley spintronics. Such a coupling suppresses the interlayer hopping, making possible the spin Hall effect and spin-dependent selection rule for optical transitions even in the presence of inversion symmetry. A static magnetic field can induce oscillations of the layer (electrical) polarization, while spin precession in magnetic field can be electrically controlled. The frequencies of the spin and layer dynamics become valley dependent when both the magnetic and electric fields are present, giving rise to beating phenomena where a spin polarization can evolve from zero net polarization. An oscillating electric field and magnetic field will both drive the hole spin resonance. Interestingly, the two fields can have a valley-dependent interference through their coupling to the same transition. Such a phenomenon not only achieves an effective coupling between the oscillating electric and magnetic fields but also makes possible a prototypical interplay between the spin and valley information carriers where quantum gates on the spin qubit are controlled by the valley bit.

\section{Results}

Strong coupling of spin and valley with layer pseudospin. Group-VI TMDC bilayers are AB stacked: one monolayer sits on another but with $180^{\circ}$ rotation (Fig. 1a). Pristine bilayers are therefore inversion symmetric. As in monolayers, $a b$ initio calculations show that the valence and conduction band edges near $K$ points in bilayers are dominantly contributed by $d_{z^{2}}, d_{x y}$, and $d_{x^{2}-y^{2}}$ orbitals of metal atoms. A minimal band model of bilayers in the neighborhood of $K$ points can be constructed by adding interlayer hopping to the $\mathbf{k} \cdot \mathbf{p}$ model of monolayers established in Xiao et al. ${ }^{20}$,

$$
H(\mathbf{q})=\left[\begin{array}{cccc}
\Delta & a t\left(\tau_{z} q_{x}+i q_{y}\right) & 0 & 0 \\
\operatorname{at}\left(\tau_{z} q_{x}-i q_{y}\right) & -\tau_{z} s_{z} \lambda & 0 & t_{\perp} \\
0 & 0 & \Delta & a t\left(\tau_{z} q_{x}-i q_{y}\right) \\
0 & t_{\perp} & \text { at }\left(\tau_{z} q_{x}+i q_{y}\right) & \tau_{z} s_{z} \lambda
\end{array}\right]
$$

The basis is $\left\{\left|d_{z^{2}}^{\mathrm{u}}\right\rangle, 1 / \sqrt{2}\left(\left|d_{x^{2}-y^{2}}^{\mathrm{u}}\right\rangle-i \tau_{z}\left|d_{x y}^{\mathrm{u}}\right\rangle\right),\left|d_{z^{2}}^{\mathrm{l}}\right\rangle\right.$, $\left.1 / \sqrt{2}\left(\left|d_{x^{2}-y^{2}}^{1}\right\rangle+i \tau_{z}\left|d_{x y}^{1}\right\rangle\right)\right\}$, where the superscripts ' $\mathrm{u}$ ' and 'l' denote the 'upper' and 'lower' layer respectively. $\mathbf{q}$ is the relative wavevector with respect to the $K$ points, $\Delta$ the monolayer band gap, $a$ the lattice constant, $t$ the nearest-neighbor intra-layer hopping, with $\lambda$ the spin-valley coupling of holes in monolayers. $t_{\perp}$ is the interlayer hopping for holes, while the interlayer hopping for electrons vanishes at $K$ points due to the symmetry of the $d_{z^{2}}$ orbital. $\tau_{z}= \pm 1$ is the valley index of bilayer bands and $s_{\mu}$ denotes the Pauli matrices for the spin. Similar to monolayers ${ }^{20}$, the spin up and spin-down states are still decoupled in bilayers, as interlayer hopping conserves spin. Table 1 lists these parameters obtained by fitting the $a b$ initio band structures.

The Hamiltonian of holes near $K$ points can be obtained through a canonical transformation of equation 1 eliminating the interband coupling,

$$
H_{\mathrm{v}}(q)=-\varepsilon_{q}-\lambda \tau_{z} \sigma_{z} s_{z}+t_{\perp} \sigma_{x},
$$

where $\varepsilon_{q}=\Delta \frac{a^{2} t^{2} q^{2}}{\Delta^{2}-\lambda^{2}}$ gives the energy dispersion. We have dropped terms with relative strength $\varepsilon_{q} / \Delta$ or smaller compared with the retained terms. $\sigma_{\mu}$ are Pauli matrices defined in the basis $\left\{\frac{1}{\sqrt{2}}\left(\left|d_{x^{2}-y^{2}}^{\mathrm{u}}\right\rangle-i \tau_{z}\left|d_{x y}^{\mathrm{u}}\right\rangle\right), \frac{1}{\sqrt{2}}\left(\left|d_{x^{2}-y^{2}}^{\mathrm{l}}\right\rangle+i \tau_{z}\left|d_{x y}^{\mathrm{l}}\right\rangle\right)\right\}, \quad$ which can be regarded as the layer pseudospin. The second term in equation 2 represents a strong coupling between the layer pseudospin $\left(\sigma_{z}\right)$, the real spin $\left(s_{z}\right)$ and the valley $\left(\tau_{z}\right)$, while the third term is the interlayer hopping. The coupling between the three indices is the manifestation of the monolayer spin-valley coupling in the AB-stacking order. This coupling is much stronger than (comparable to) the interlayer hopping in $\mathrm{WX}_{2}$ $\left(\mathrm{MoX}_{2}\right)$ as listed in Table 1 , and it originates from the strong spin-orbit interaction in the $d$ orbitals of the metal atoms. A direct consequence is that the interlayer hopping is virtually suppressed as observed in $\mathrm{WX}_{2}$ multilayers ${ }^{25}$.

At each wavevector $\mathbf{q}$, the eigenstates of equation 2 are two spin doublets separated by a large energy $2 \sqrt{\lambda^{2}+t_{\perp}^{2}}$. We focus on the spin doublet at the band edge (enclosed by the dashed box in Fig. 1c), which in valley $K$ is given by

$$
\begin{aligned}
& |K, \uparrow\rangle=\frac{1}{\sqrt{2}}\left(\sin \alpha\left(\left|d_{x^{2}-y^{2}}^{\mathrm{u}}\right\rangle-i\left|d_{x y}^{\mathrm{u}}\right\rangle\right)+\cos \alpha\left(\left|d_{x^{2}-y^{2}}^{\mathrm{l}}\right\rangle+i\left|d_{x y}^{\mathrm{l}}\right\rangle\right)\right) \otimes|\uparrow\rangle, \\
& |K, \downarrow\rangle=\frac{1}{\sqrt{2}}\left(\cos \alpha\left(\left|d_{x^{2}-y^{2}}^{\mathrm{u}}\right\rangle-i\left|d_{x y}^{\mathrm{u}}\right\rangle\right)+\sin \alpha\left(\left|d_{x^{2}-y^{2}}^{\mathrm{u}}\right\rangle+i\left|d_{x y}^{\mathrm{l}}\right\rangle\right)\right) \otimes|\downarrow\rangle .
\end{aligned}
$$

$|\uparrow\rangle$ and $|\downarrow\rangle$ denote the spin up and down states, respectively. The spin doublet $\{|\bar{K}, \downarrow\rangle,|\bar{K}, \uparrow\rangle\}$ in valley $-K$ is the time reversal of the above. These energy eigenstates are associated with a spinand valley-dependent layer (electrical) polarization,

$$
\left\langle\sigma_{z}\right\rangle=-s_{z} \tau_{z} \cos 2 \alpha, \quad \cos 2 \alpha \equiv \frac{\lambda}{\sqrt{\lambda^{2}+t_{\perp}^{2}}},
$$

Table 1 lists the computed values of $\cos 2 \alpha$ using the parameters $t_{\perp}$ and $\lambda$ fitted from the $a b$ initio band dispersion, which agrees well with the layer polarization directly evaluated from the $a b$ initio wavefunction. This shows that our minimal model gives a good description of the electronic states near $K$ points. The large layer polarization means that the spin doublet is predominantly localized in (opposite) individual layers, and interlayer hopping of holes is largely suppressed. We note that at equilibrium, the four 
a

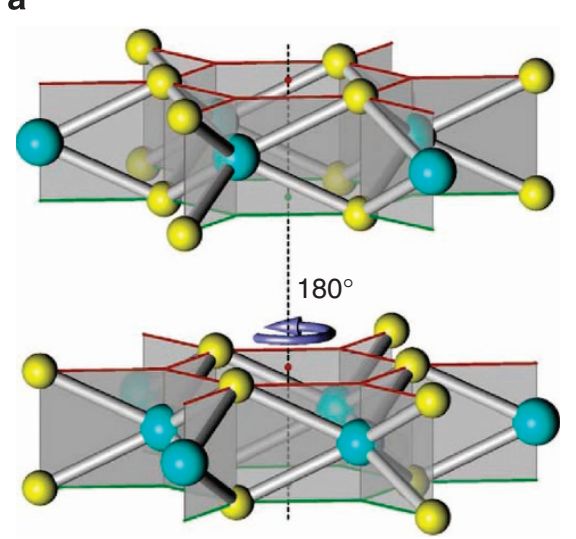

c

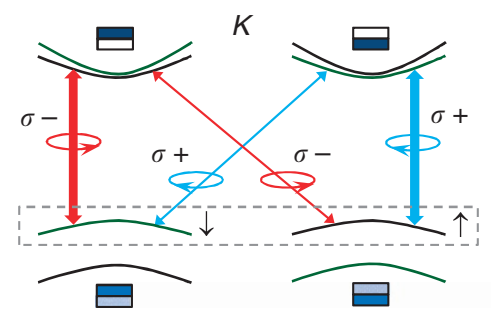

b
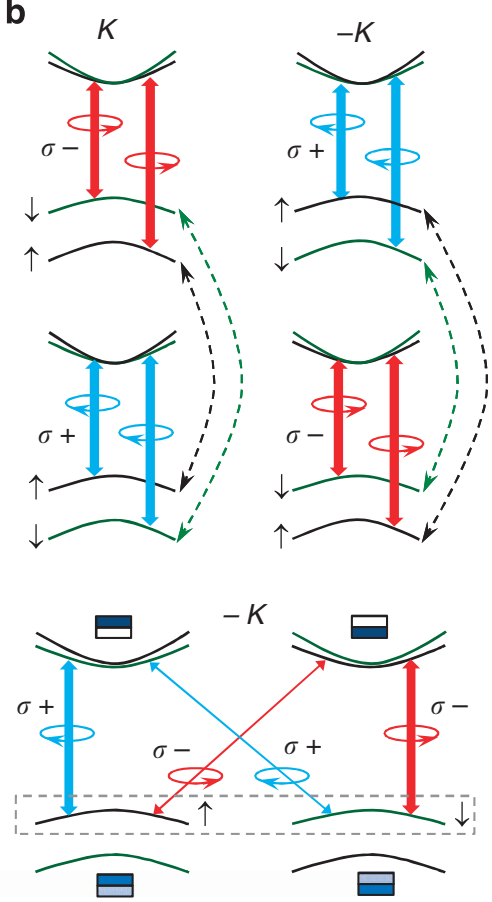

Figure 1 | Group-VI TMDC bilayer. (a) Coordination structure of AB-stacked bilayer. (b) Optical transition selection rules in decoupled monolayers in AB-stacking order. Dashed arrows indicate interlayer hopping. (c) Optical transition selection rules in the $K$ and $-K$ valleys of bilayers with interlayer hopping. The transitions denoted by the thick (thin) arrows have strength $\propto \cos 2 \alpha(\propto \sin 2 \alpha$ ). The layer polarization of the Bloch states is schematically illustrated with the rectangular blocks where darker color denotes more occupation.

Table 1 | Fitting result from $a b$ initio band structure calculations.

\begin{tabular}{|c|c|c|c|c|c|c|c|}
\hline & $a(\AA)$ & $\Delta$ & $t$ & $\mathbf{2} \mathbf{t}_{\perp}$ & $2 \lambda$ & $\cos 2 \alpha$ & $\left\langle\boldsymbol{\sigma}_{\mathbf{z}}\right\rangle_{\mathbf{K}}$ \\
\hline $\mathrm{MoS}_{2}$ & 3.160 & 1.766 & 1.137 & 0.086 & 0.147 & 0.863 & 0.863 \\
\hline $\mathrm{WS}_{2}$ & 3.153 & 1.961 & 1.436 & 0.109 & 0.421 & 0.968 & 0.962 \\
\hline $\mathrm{MoSe}_{2}$ & 3.288 & 1.541 & 0.951 & 0.106 & 0.182 & 0.864 & 0.855 \\
\hline $\mathrm{WSe}_{2}$ & 3.280 & 1.698 & 1.233 & 0.134 & 0.456 & 0.959 & 0.944 \\
\hline
\end{tabular}

$\Delta, t$, and $\lambda$ (in units of electron volt) are fitted from the monolayer band structures. Interlayer hopping $t_{\perp}$ is read out from the valence band splitting at $K$ points of bilayers in the absence of spin-orbit coupling. The structural constants use the bulk values. The column $\cos 2 \alpha$ is evaluated using equation 4 with the listed values of $t_{\perp}$ and $\lambda$, and according to our band model it gives the layer polarization of the Bloch state $|K \downarrow\rangle$. The last column is the layer polarization directly evaluated from the ab initio wavefunction of $|K \downarrow\rangle$. See also Supplementary Note 1.

states $|K, \downarrow\rangle,|K, \uparrow\rangle,|\bar{K}, \downarrow\rangle$ and $|\bar{K}, \uparrow\rangle$ are equally populated and there is no net layer (electrical) polarization.

An important consequence of the spin-dependent layer polarization is that bilayers will inherit most of the spin physics of monolayers. In Fig. 1c, we illustrate the optical interband transitions by circularly polarized light between the valence and conduction band edges near $K$ points. Spin is conserved here by the optical transition. In both valleys, $\sigma+(\sigma-)$ polarized light predominantly excites spin up (down) photocarriers ${ }^{22}$. We find the degree of spin polarization created upon absorption of a circularly polarized photon to be $\cos 2 \alpha$, directly corresponding to the layer polarization of the hole states. This spin-dependent selection rule can be used for optical injection and detection of spin polarization near $K$ points. The valley circular dichroism is absent in pristine bilayers because of inversion symmetry. Moreover, the Berry curvature of the band edge holes at $K$ points is:

$$
\Omega=s_{z} \frac{2 a^{2} t^{2}}{\left(\Delta-\sqrt{\lambda^{2}+t_{\perp}^{2}}\right)^{2}} \cos 2 \alpha,
$$

which depends only on the spin index. Thus, the spin Hall effect of holes is also present in inversion symmetric bilayers, while the valley Hall effect vanishes.

Magnetoelectric effects. While the spin couples to real space magnetic fields, the layer (electrical) polarization couples to electric fields in the perpendicular $(z)$ direction. The coupling between spin and layer pseudospin therefore gives rise to magnetoelectric effects. The hole Hamiltonian in the presence of electromagnetic fields is,

$$
H_{\mathrm{v}}=-\lambda \tau_{z} \sigma_{z} s_{z}+t_{\perp} \sigma_{x}+B_{x} s_{x}+B_{z} s_{z}+E_{z} \sigma_{z} .
$$

Without loss of generality, the in-plane component of the magnetic field is taken to be along the $x$-direction. $B_{x}, B_{\mathrm{z}}$ and $E_{z}$ are strengths of magnetic and electric fields in units of energy, normalized by the magnetic and electric dipole respectively. $B_{x}$ and $B_{z}$ can be up to $\sim$ millielectronvolts for magnetic fields up to a few Tesla. A moderate electric field is considered where $E_{z}$ has comparable magnitude to $B_{x}$ and $B_{\mathrm{z}}$, so $\lambda, t_{\perp}>>B_{x}, B_{\mathrm{z}}, E_{z}$. We focus on the spin doublet at the band edge (c.f. equation 3 ), and 


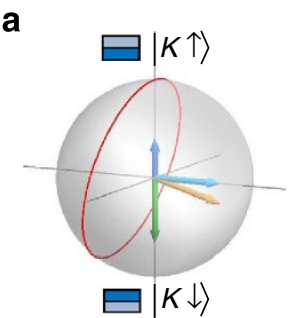

$K$

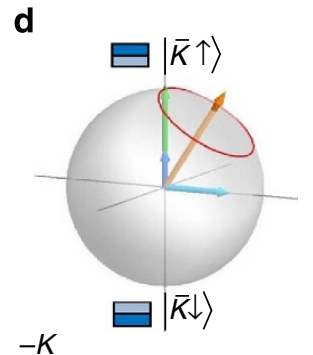

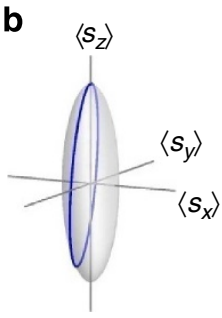
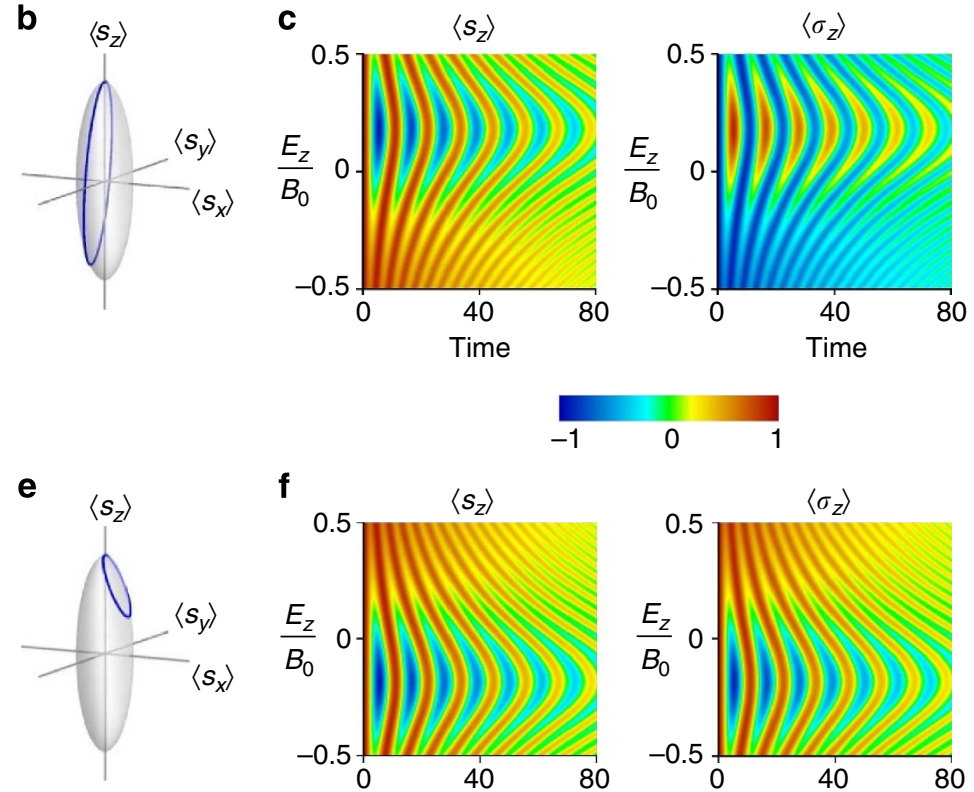
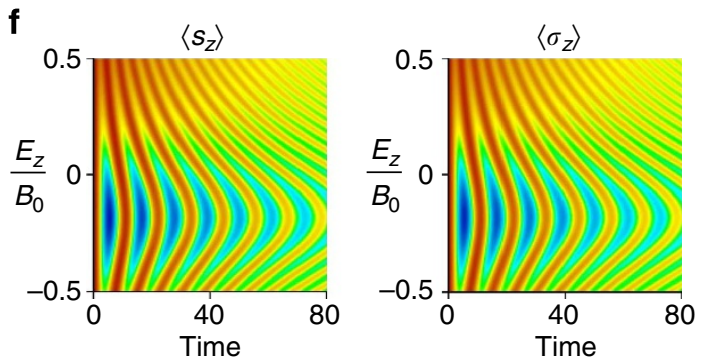

Figure 2 | Valley-dependent oscillations of spin and layer polarizations. (a) Bloch sphere spanned by superposition states of the doublet $\{|K \uparrow\rangle, \mid K \downarrow\}$. Dark and light blue arrows denote the effective fields from the magnetic field components $B_{z}$ and $B_{x}$ respectively, and green arrow denotes that from the electric field $E_{z}$. Yellow arrow is the total effective field. (b) Anomalous precessions of the spin vector $\mathbf{s}$ when layer is traced out. (c) Oscillations of the spin $\left(\left\langle s_{z}\right\rangle\right)$ and layer $\left(\left\langle\sigma_{z}\right\rangle\right)$ polarizations in magnetic field $\left(B_{x}, B_{z}\right)=\left(B_{0} \cos 10^{\circ}, B_{0} \sin 10^{\circ}\right)$, from the initial state $|K \uparrow\rangle$. The oscillations are electrically tunable. (d-f) Same plots in valley $-K$. The unit of time is $B_{0}^{-1}$.

the Hamiltonian in equation 6 projected into the subspace of this doublet reads,

$$
H_{\mathrm{D}}=\left[\begin{array}{cc}
-\tau_{z} E_{z} \cos 2 \alpha+B_{z} & B_{x} \sin 2 \alpha \\
B_{x} \sin 2 \alpha & \tau_{z} E_{z} \cos 2 \alpha-B_{z}
\end{array}\right]
$$

where the basis is $\{|K \uparrow\rangle,|K \downarrow\rangle\}$ at valley $K\left(\tau_{z}=1\right)$ and $\{|\bar{K} \uparrow\rangle,|\bar{K} \downarrow\rangle\}$ at valley $-K\left(\tau_{z}=-1\right)$. The effective fields are illustrated in Fig. 2a,d for valley $K$ and $-K$, respectively.

As the doublet is associated with spin- and valley-dependent layer (electrical) polarization, its coupling to both the electric and magnetic fields gives rise to various forms of magnetoelectric effects. A few examples are given. Figure 3 a shows that spin precessions in a magnetic field can be controlled by the electric field $E_{z}$. Here the initial state is a spin polarized one with no layer and valley polarizations: $\rho_{\mathrm{s}}=0.5|K \uparrow\rangle\langle K \uparrow|+0.5| \bar{K} \uparrow\rangle\langle\bar{K} \uparrow|$. Figure $3 \mathrm{~b}$ shows that static magnetic field with finite in-plane component can induce oscillations of the electrical polarization $\left\langle\sigma_{z}\right\rangle$. The initial state is a layer-polarized one with no spin and valley polarizations: $\rho_{\mathrm{E}}=0.5|K \uparrow\rangle\langle K \uparrow|+0.5| \bar{K} \downarrow\rangle\langle\bar{K} \downarrow|$. We note that spin polarization can be prepared by optical pumping with circularly polarized light (c.f. Fig. 1c), while layer polarization can be prepared by tunneling from hole reservoir at one side (e.g., the lower side) of the bilayer as interlayer hopping is virtually suppressed.

When both $E_{z}$ and $B_{z}$ are finite, the direction and the magnitude of the total effective field become valley dependent (c.f. equation 7), resulting in different oscillation frequencies in the two valleys as illustrated in Fig. 2. This gives rise to beating phenomena in the oscillation of spin and electrical polarizations when both valleys are populated, as shown in Fig. 3. Remarkably, because of the beating, a finite spin polarization can evolve out of an initial state with zero spin polarization in all directions (Fig. 3c).

We note that an arbitrary linear superposition of the doublet is in fact an entangled state between the spin and the layer pseudospin as shown in equation 3. After tracing out the layer degrees of freedom, the magnitude of the in-plane spin component will scale down by a factor of $\sin 2 \alpha$, and the spin vector always lies within an elliptical sphere (c.f. Fig. 2b,e).

Valley-controlled quantum gate of spin qubit. When static electric field $E_{z}$ or magnetic field $B_{z}$ is applied to split the doublet along the $z$ axis, an oscillating in-plane magnetic field $B_{x}$ can drive the hole spin resonance. The doublet splitting becomes valley dependent when both $E_{z}$ and $B_{z}$ are finite (c.f. equation 7 and Fig. 4a). Thus, the hole spin resonance can be selectively addressed at valley $K$ or $-K$ by choosing the frequency of $B_{x}$.

As a quantum two-level system, the doublet can be used as a spin qubit for information processing. The valley index of the doublet, on the other hand, represents another bit of information. A prototype interplay between the two types of information carriers for potential valley-spintronic applications can be made possible. For example, as shown in Fig. 4a, we consider the application of a pulsed $B_{x}$ with central frequency on resonance with the splitting in valley $-K$. If the valley bit is in state $-K$, the spin qubit is coherently rotated about the $x$ axis by the pulse. If the valley bit is in state $K$, the spin qubit is unchanged because of the large detuning. This valley-dependent rotation represents a quantum gate on the spin qubit controlled by the valley bit. If the rotation is $\pi$, a valley-controlled NOT gate on the spin qubit is realized. We note that this controlled NOT gate can be used for deterministic conversion between an electrical polarization and a spin polarization (for example, between $\rho_{\mathrm{E}}$ and $\rho_{\mathrm{s}}$ ).

Interference between oscillating electric and magnetic fields. If a static $B_{x}$ is applied to split the doublet along the $x$ axis, both the oscillating electric field and magnetic field in the $z$ direction can drive the hole spin resonance ${ }^{26-30}$. Pulsed oscillating $E_{z}$ or $B_{z}$ can therefore realize coherent rotations of the spin qubit about the $z$ axis. Interestingly, interference between the oscillating electric field and magnetic field can now be realized through their 

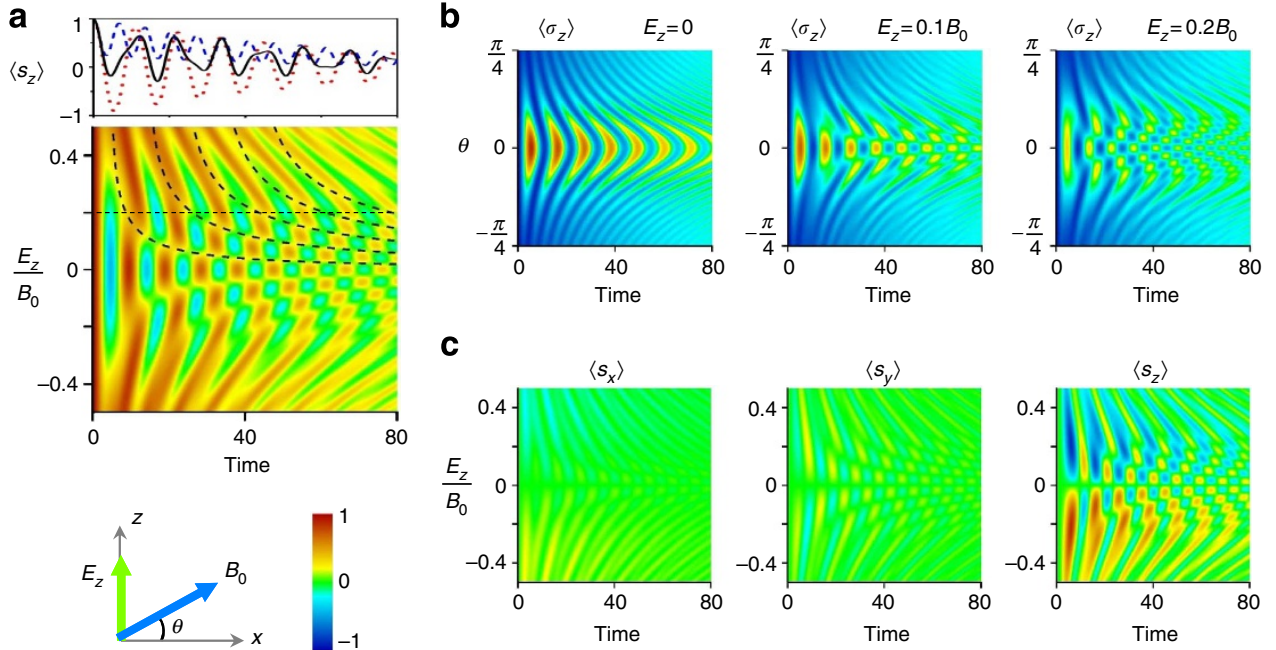

Figure 3 | Magnetoelectric effects. (a) Spin precession in magnetic field along $\theta=10^{\circ}$ (c.f. inset), from initial state $\rho_{\mathrm{s}}=0.5|\mathrm{~K} \uparrow\rangle\langle\mathrm{K} \uparrow|+0.5| \overline{\mathrm{K}} \uparrow\rangle\langle\overline{\mathrm{K}} \uparrow|$. Oscillation in $\left\langle s_{z}\right\rangle$ is tunable by the electric field $E_{z}$ as shown by the contour plot. The dashed curves on the contour image denote the time when the spin precessions at $K$ and $-K$ acquire a phase difference of $(2 n+1) \pi$. A horizontal cut of the contour image at $E_{z}=0.2 B_{0}$ is shown as the black solid curve atop, while the red and blue curves denote $\left\langle s_{z}\right\rangle$ at $K$ and $-K$, respectively. (b) Oscillation of the layer polarization $\left\langle\sigma_{z}\right\rangle$ in a magnetic field, with a pattern tunable by $E_{z}$. The initial state is $\rho_{\mathrm{E}}=0.5|\mathrm{~K} \uparrow\rangle\langle\mathrm{K} \uparrow|+0.5| \overline{\mathrm{K}} \downarrow\rangle\langle\overline{\mathrm{K}} \downarrow|$. (c) Finite spin polarization can evolve out of the initial state $\rho_{\mathrm{E}}$ of zero spin polarization. Magnetic field is along the direction $\theta=10^{\circ}$. In all calculations, we assumed the doublet has a spin relaxation rate of $0.01 B_{0}$. The unit of time is $B_{0}^{-1}$.
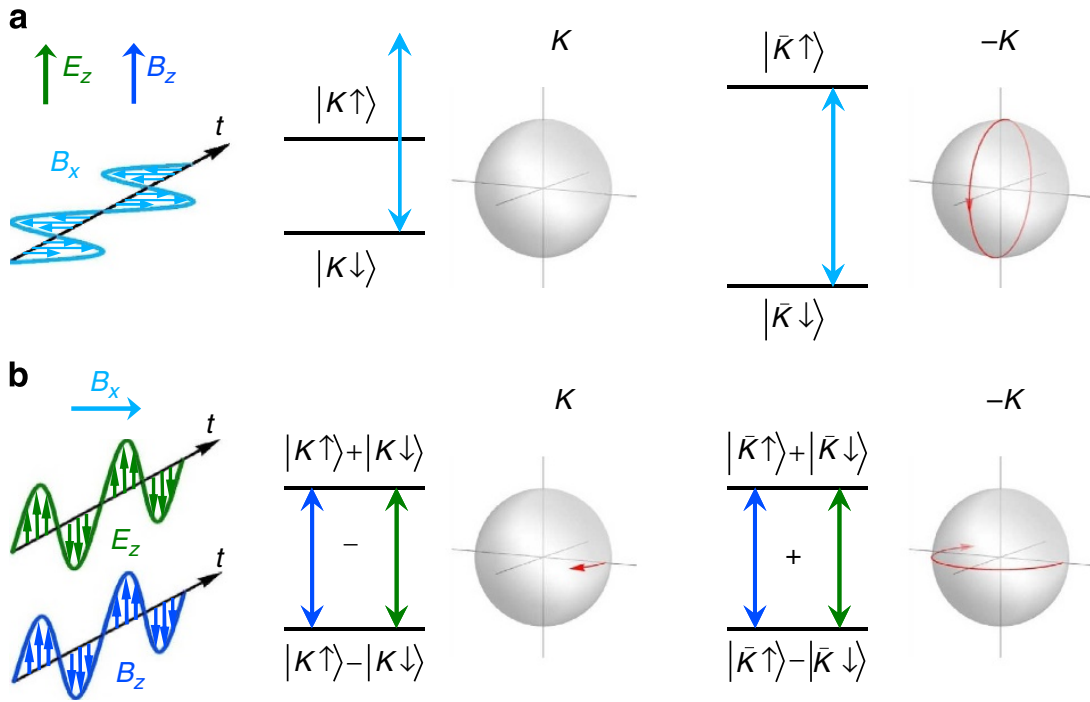

Figure 4 | Valley-controlled quantum gates of a spin qubit. (a) The Zeeman splitting of the spin qubit has different magnitude in valley $K$ and $-K$ in presence of both static electric $\left(E_{z}\right)$ and magnetic $\left(B_{z}\right)$ fields. A pulse of oscillating magnetic field $B_{x}$ in resonance with the splitting at $-K$ rotates the spin qubit only if the valley bit is on state $-K$, as illustrated on the spin Bloch sphere. (b) When a static $B_{x}$ splits the spin qubit, both oscillating $E_{z}$ and $B_{z}$ can drive the spin resonance, and the two fields constructively (destructively) interfere in valley $-K(K)$, which realize valley-controlled rotation of the spin qubit about the $z$ axis.

coupling to the same transition. The interference is valley dependent, as the coupling of the spin qubit to $E_{z}$ has a valleydependent sign. If $E_{z}(t)$ and $B_{z}(t)$ are in phase with the same frequency, their interference is destructive in valley $K$ and constructive in valley $-K$. Such a phenomenon induces an effective coupling between the oscillating electric field and magnetic field in the presence of valley polarization. The valleydependent interference can also be utilized for valley-controlled spin qubit rotations about the $z$ axis, as shown in Fig. $4 \mathrm{~b}$.

\section{Discussion}

The phenomena above are predicted for holes in the $K$ valleys. Holes can be optically injected into $K$ valleys in group-VI TMDC bilayers by pumping the direct transition between the higherlying valence band and the conduction band. The strong PL peak associated with this transition suggests high efficiency of the injection $21,22,24,25$. Interestingly, ab initio calculations show that although $\mathrm{WSe}_{2}$ bilayer has an indirect gap, the valence band maxima are at the $K$ points. For $\mathrm{MoSe}_{2}$ and $\mathrm{WS}_{2}$ bilayers, hole pockets are also expected to appear at $K$ points at low doping (Supplementary Note 1). Thus, lightly p-doped $\mathrm{WSe}_{2}, \mathrm{MoSe}_{2}$ and $\mathrm{WS}_{2}$ bilayers could be ideal platforms to explore these phenomena.

Long lifetimes of the polarizations are also essential for valleyspintronic applications. Spin and valley relaxations in TMDC bilayers can be qualitatively different from those in monolayers ${ }^{31}$, 
and remain open questions. Notably, for holes near $K$ points, spin flip $(|K \uparrow\rangle \leftrightarrow|K \downarrow\rangle)$ and valley flip $(|K \uparrow\rangle \leftrightarrow|\bar{K} \uparrow\rangle)$ are suppressed by the spin- and valley-dependent layer polarization from the coupling of the three indices. As $|K \downarrow\rangle$ and $|\bar{K} \uparrow\rangle$ are located on the layer opposite to $|K \uparrow\rangle$, the spatial overlap of the initial and final state wavefunctions of spin and valley flips is small, which is given by $\sin ^{2} 2 \alpha \sim 0.1(\sim 0.3)$ in $\mathrm{WX}_{2}\left(\operatorname{MoX}_{2}\right)$ bilayers. This small factor helps to reduce both spin and the valley relaxations. Moreover, long valley lifetime has also been suggested by the observation of valley polarization in biased $\mathrm{MoS}_{2}$ bilayers $^{24}$.

Finally, we note that the form of effective coupling here between spin, valley and layer pseudospin is dictated by the symmetries. The absence of inversion symmetry and the presence of mirror symmetry plus time-reversal symmetry in monolayers dictate the spin-valley coupling in the form of $\tau_{z} s_{z}$ (ref. 20). The $\mathrm{AB}$-stacking order in bilayer also dictates the layer-dependent sign of this spin-valley coupling. These two facts combined together determine the spin-orbit coupling to be $\sigma_{z} \tau_{z} s_{z}$, which observes the inversion symmetry of AB-stacked bilayers. The interplay between this coupling and the interlayer hopping gives rise to the predicted magetoelectric effects, which may also be present in other layered systems with the above symmetry properties and pronounced spin-orbit coupling.

\section{Methods}

$\boldsymbol{A} \boldsymbol{b}$ initio calculations. $A b$ initio calculations were performed using the density functional theory in conjunction with all-electron projector augmented wave (PAW) potentials ${ }^{32,33}$ and the Perdew-Burke-Ernzerhof ${ }^{34}$ generalized gradient approximation exchange-correlation potentials as implemented in ABINIT package ${ }^{35,36}$. Energy cutoff ecut $=380 \mathrm{eV}$ and pawecutdg $=750 \mathrm{eV}$ were used and convergence criteria of total energy was $10^{-4} \mathrm{eV}$. Vacuum layer thickness of more than $15 \AA$ in $z$ direction was used for all bilayers. Gamma-centered k-mesh $20 \times 20 \times 1$ was used. Spin-orbit coupling (SOC) was accounted for in our calculations. Wavefunctions of $\mathrm{MoX}_{2}$ and $\mathrm{WX}_{2}(\mathrm{X}=\mathrm{S}$ or Se) for valence top at $K$ were extracted for the calculations of layer polarization (Table 1).

\section{References}

1. Insight: Spintronics. Nat. Mater. 11, 367-416 (2012).

2. Hanson, R., Kouwenhoven, L. P., Petta, J. R., Tarucha, S. \& Vandersypen, L. M. K. Spins in few-electron quantum dots. Rev. Mod. Phys. 79, 1217-1265 (2007).

3. Liu, R. B., Yao, W. \& Sham, L. J. Quantum computing by optical control of electron spins. Adv. Phys. 59, 703-802 (2010).

4. Novoselov, K. S. et al. Electric field effect in atomically thin carbon films. Science 306, 666-669 (2004)

5. Novoselov, K. S. et al. Two-dimensional gas of massless Dirac fermions in graphene. Nature 438, 197-200 (2005).

6. Zhang, Y. B., Tan, Y. -W., Stormer, H. L. \& Kim, P. Experimental observation of the quantum Hall effect and Berry's phase in graphene. Nature 438, 201-204 (2005).

7. Novoselov, K. S. et al. Two-dimensional atomic crystals. Proc. Natl Acad. Sci. USA 102, 10451-10453 (2005).

8. Wang, Q. H., Kalantar-Zadeh, K., Kis, A., Coleman, J. N. \& Strano, M. S. Electronics and optoelectronics of two-dimensional transition metal dichalcogenides. Nat. Nanotechnol. 7, 699-712 (2012).

9. Mak, K. F., Lee, C. -G., Hone, J., Shan, J. \& Heinz, T. F. Atomically thin $\mathrm{MoS}_{2}$ : a new direct-gap semiconductor. Phys. Rev. Lett. 105, 136805 (2010).

10. Splendiani, A. et al. Emerging photoluminescence in monolayer $\mathrm{MoS}_{2}$. Nano Lett. 10, 1271-1275 (2010).

11. San-Jose, P., Prada, E., McCann, E. \& Schomerus, H. Pseudospin valve in bilayer graphene: towards graphene-based pseudospintronics. Phys. Rev. Lett. 102, 247204 (2009).

12. Min, H. K., Borghi, G., Polini, M. \& MacDonald, A. H. Pseudospin magnetism in graphene. Phys. Rev. B 77, 041407(R) (2008).

13. Banerjee, S. K., Register, L. F., Tutuc, E., Reddy, D. \& MacDonald, A. H. Bilayer pseudospin field-effect transistor (bisfet): a proposed new logic device. IEEE Electron. Device Lett. 30, 158-160 (2009).

14. Pesin, D. \& MacDonald, A. H. Spintronics and pseudospintronics in graphene and topological insulators. Nat. Mater. 11, 409-416 (2012).

15. Gunawan, O., Shkolnikov, Y. P., Vakili, K., Gokmen, T., De Poortere, E. P. \& Shayegan, M. Valley susceptibility of an interacting two-dimensional electron system. Phys. Rev. Lett. 97, 186404 (2006).
16. Rycerz, A., Tworzydlo, J. \& Beenakker, C. W. J. Valley filter and valley valve in graphene. Nat. Phys. 3, 172-175 (2007).

17. Xiao, D., Yao, W. \& Niu, Q. Valley-contrasting physics in graphene: magnetic moment and topological transport. Phys. Rev. Lett. 99, 236809 (2007).

18. Yao, W., Xiao, D. \& Niu, Q. Valley-dependent optoelectronics from inversion symmetry breaking. Phys. Rev. B 77, 235406 (2008).

19. Zhu, Z. -W., Collaudin, A., Fauque, B., Kang, W. \& Behnia, K. Field-induced polarization of Dirac valleys in bismuth. Nat. Phys. 8, 89-94 (2012).

20. Xiao, D., Liu, G. -B., Feng, W. -X., Xu, X. -D. \& Yao, W. Coupled spin and valley physics in monolayers of $\mathrm{MoS}_{2}$ and other group-VI dichalcogenides. Phys. Rev. Lett. 108, 196802 (2012).

21. Zeng, H. L., Dai, J. F., Yao, W., Xiao, D. \& Cui, X. D. Valley polarization in $\mathrm{MoS}_{2}$ monolayers byoptical pumping. Nat. Nanotechnol. 7, 490-493 (2012).

22. Mak, K. F., He, K., Shan, J. \& Heinz, T. F. Control of valley polarization in monolayer $\mathrm{MoS}_{2}$ by optical helicity. Nat. Nanotechnol. 7, 494-498 (2012).

23. Cao, T. et al. Valley-selective circular dichroism of monolayer molybdenum disulphide. Nat. Commun. 3, 887 (2012).

24 . Wu, S. et al. Electrical tuning of valley magnetic moment via symmetry control. Nat. Phys. 9, 149-153 (2013).

25. Zeng, H. et al. Optical signature of symmetry variations and spin-valley coupling in atomically thin tungsten dichalcogenides. Sci. Rep. 3, 1608 (2013).

26. Salis, G., Kato, Y., Ensslin, K., Driscoll, D. C., Gossard, A. C. \& Awschalom, D. D. Electrical control of spin coherence insemiconductor nanostructures. Nature 414, 619-622 (2001).

27. Kato, Y., Myers, R. C., Driscoll, D. C., Gossard, A. C., Levy, J. \& Awschalom, D. D. Gigahertz electron spin manipulation using voltage-controlled g-tensor modulation. Science 299, 1201-1204 (2003).

28. Schulte, M., Lok, J. G. S., Denninger, G. \& Dietsche, W. Electron spin resonance on a two-dimensional electron gas in a single alas quantum well. Phys. Rev. Lett. 94, 137601 (2005).

29. Nowack, K. C., Koppens, F. H. L., Nazarov, Y. V. \& Vandersypen, L. M. K. Coherent control of a single electron spin with electric fields. Science $\mathbf{3 1 8}$, 1430-1433 (2007).

30. Nadj-Perge, S., Frolov, S. M., Bakkers, E. \& Kouwenhoven, L. P. Spin-orbit qubit in a semiconductor nanowire. Nature 468, 1084-1087 (2010).

31. Song, Y. \& Dery, H. Symmetry based transport theory of monolayer transitionmetal dichalcogenides. Preprint at http://arxiv.org/abs/1302.3627 (2013).

32. Bloöchl, P. E. Projector augmented-wave method. Phys. Rev. B 50, 17953-17979 (1994).

33. Torrent, M., Jollet, F., Bottin, F., Zérah, G. \& Gonze, X. Implementation of the projector augmented-wave method in the ABINIT code: application to the study of iron under pressure. Comput. Mater. Sci. 42, 337-351 (2008).

34. Perdew, J. P., Burke, K. \& Ernzerhof, M. Generalized gradient approximation made simple. Phys. Rev. Lett. 77, 3865-3868 (1996).

35. Gonze, X. A brief introduction to the ABINIT software package. Z. Kristallogr. 220, 558-562 (2005).

36. Gonze, X. et al. First-principles computation of material properties: the ABINIT software project. Comput. Mater. Sci. 25, 478-492 (2002).

\section{Acknowledgements}

We thank A.M. Jones for proof reading. This work is mainly supported by the Research Grant Council (HKU 706412 P) and the University Grant Council (AoE/P-04/08) of Hong Kong SAR. X.X. is supported by US DoE, BES, Materials Sciences and Engineering Division (DE-SC0008145). D.X. is supported by US DoE, BES, Materials Sciences and Engineering Division.

\section{Author contributions}

W.Y. conceived and designed the research, with inspiration from X.X., X.C. and D.X. Z.G., G.-B.L., H.Y. and W.Y. performed the calculations and analysis. W.Y., G.-B.L., Z.G. and X.X. wrote the manuscript. All authors discussed the results and commented on the manuscript.

\section{Additional information}

Supplementary Information accompanies this paper at http://www.nature.com/ naturecommunications

Competing financial interests: The authors declare no competing financial interests.

Reprints and permission information is available online at http://npg.nature.com/ reprintsandpermissions/

How to cite this article: Gong, Z. et al. Magnetoelectric effects and valley-controlled spin quantum gates in transition metal dichalcogenide bilayers. Nat. Commun. 4:2053 doi: $10.1038 /$ ncomms3053 (2013). 